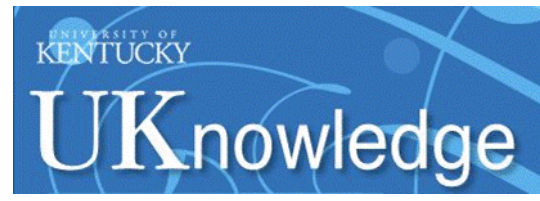

University of Kentucky

UKnowledge

$11-22-2017$

\title{
Transient Analysis of Fracture Initiation in a Coupled Thermoelastic Solid
}

Louis Milton Brock

University of Kentucky, louis.brock@uky.edu

Follow this and additional works at: https://uknowledge.uky.edu/me_facpub

Part of the Mechanical Engineering Commons, and the Structures and Materials Commons Right click to open a feedback form in a new tab to let us know how this document benefits you.

\section{Repository Citation}

Brock, Louis Milton, "Transient Analysis of Fracture Initiation in a Coupled Thermoelastic Solid" (2017). Mechanical Engineering Faculty Publications. 45.

https://uknowledge.uky.edu/me_facpub/45

This Article is brought to you for free and open access by the Mechanical Engineering at UKnowledge. It has been accepted for inclusion in Mechanical Engineering Faculty Publications by an authorized administrator of UKnowledge. For more information, please contact UKnowledge@lsv.uky.edu. 


\section{Transient Analysis of Fracture Initiation in a Coupled Thermoelastic Solid \\ Digital Object Identifier (DOI)}

https://doi.org/10.2140/jomms.2017.12.667

Notes/Citation Information

Published in Journal of Mechanics of Materials and Structures, v. 12, no. 5, p. 667-688.

The publisher has granted the permission for posting the article here.

This article is available at UKnowledge: https://uknowledge.uky.edu/me_facpub/45 


\title{
TRANSIENT ANALYSIS OF FRACTURE INITIATION IN A COUPLED THERMOELASTIC SOLID
}

\author{
LOUIS M. BROCK
}

\begin{abstract}
An isotropic, thermoelastic solid is initially at rest at uniform (absolute) temperature, and contains a semi-infinite, plane crack. Application of in-plane and normal point forces to each face of the crack causes transient 3D growth. The related problem of discontinuities in temperature and displacement that exist on regions that exhibit dynamic similarity is first considered. Asymptotic expressions, whose inverses are valid near the crack edges for short times, are obtained in integral transform space. These lead to equations of the Wiener-Hopf type for the fracture problem. Analytical solutions are obtained and, upon inversion, subjected to a dynamic energy release rate criterion that accounts for kinetic energy. A particular form of rapid growth in time of the forces is found to cause crack initiation growth rates that indeed vary with position, but not with time. The influence of particular types of mixed-mode loading upon crack edge contour and thermal response near the edge is also examined.
\end{abstract}

\section{Introduction}

Crack edge location in a transient 3D study is defined by a (possibly nonrectilinear) contour in the crack plane. The semi-infinite, planar crack in an unbounded isothermal solid is treated in [Brock 2017a]. Fracture is driven by mixed-mode, point force loading on the crack faces. The dynamic energy release rate criterion [Freund 1972; 1990] is imposed, but with kinetic energy taken into account [Gdoutos 1993]. It is found that a particular time history for the loading can generate a crack edge contour that varies with position, but not with time. The solution process begins by considering the related problem of displacement discontinuity generation on a portion of a planar surface in the solid. A set of three equations is generated that involve six integral transform functions (three components of discontinuity, three components of planar surface traction). However, the set can be rewritten as three equations of the Wiener-Hopf type [Morse and Feshbach 1953]. Exact solutions are possible, and upon inversion they lead to a nonlinear differential equation for the crack edge contour.

The corresponding problem for the coupled thermoelastic solid is treated in [Brock 2017b]. Crack initiation is the focus, so that:

(a) thermal relaxation [Ignaczak and Ostoja-Starzewski 2010] can be important, and

(b) asymptotic forms of the governing equations for thermal relaxation are viable.

The results are similar in nature to those in [Brock 2017a], with the proviso that response is valid for short times.

Keywords: thermoelastic, relaxation, transient, fracture, kinetic energy, crack contour. 
However, as a first step the possibility of discontinuity in temperature across the crack gap is ignored in [Brock 2017b]. A consequence is that the solution process again involves three equations of the WienerHopf type in transform space. In the present article, the possibility of temperature discontinuity is treated. It will be seen that the solution process must now deal with a set of four equations, with integral transforms for the temperature discontinuity and heat flux across the planar surface involved. Two of the WienerHopf equations now yield analytical expressions whose inverses are valid only near the crack edge. However, imposition of the energy release rate criterion [Freund 1972; 1990] in [Brock 2017a; 2017b], and subsequent analysis of crack edge contour, actually involve the use of such asymptotic expressions. Therefore several key results of the present article can be compared with those of [Brock 2017b].

\section{Problem statement}

A closed crack $A_{C}\left(x_{3}^{0}=0, x_{1}^{0}<0\right)$ with boundary $C\left(x_{1}^{0}, x_{3}^{0}\right)=0$ exists in an unbounded, coupled thermoelastic, solid. Cartesian coordinates $\boldsymbol{x}_{0}=\boldsymbol{x}_{0}\left(x_{k}^{0}\right), k=(1,2,3)$ are used. The solid is at rest for time $t \leq 0$ at (absolute) uniform temperature $T_{0}$. For $t>0$ point forces (both shear and compressive) appear on both crack faces at $\left(x_{1}^{0}=0-, x_{2}^{0}=0, x_{3}^{0}=0 \pm\right)$. Brittle fracture is instantaneous, and the crack extends outward from $x_{0}=0$. The crack now occupies region $A_{C}+\delta A$. Boundary $C$ now includes a concave bulge:

$$
\begin{gathered}
\sqrt{\left(x_{1}^{0}\right)^{2}+\left(x_{2}^{0}\right)^{2}}=l(\psi, t), \quad l(\psi, t)=V(\psi) t, \\
0<V<V_{R}, \quad \psi=\tan ^{-1} \frac{x_{2}^{0}}{x_{1}^{0}}(|\psi|<\pi / 2) .
\end{gathered}
$$

Equation (1) implies a dynamically similar fracture process, and requires that (speed parameter) $V$ not exceed Rayleigh value $V_{R}$. Displacement $\boldsymbol{u}\left(u_{k}\right)$, traction $\boldsymbol{T}\left(\sigma_{i k}\right)$ and $\theta$, the change in temperature from $T_{0}$, are field variables. For the Lord and Shulman thermal relaxation model [Lord and Shulman 1967; Brock 2009; Ignaczak and Ostoja-Starzewski 2010]:

$$
\begin{aligned}
\nabla \cdot \boldsymbol{T}-\rho D^{2} \boldsymbol{u} & =0, \\
\left(k_{T} \nabla^{2}-\rho C_{E} \mathrm{P} D\right) \theta+\mu \alpha_{D} T_{0} \mathrm{P} D(\nabla \cdot \boldsymbol{u}) & =0, \\
\frac{1}{\mu} \boldsymbol{T}=\left[\frac{2 v}{1-2 v}(\nabla \cdot \boldsymbol{u}) \boldsymbol{1}-\alpha_{D} \theta\right]+\nabla \boldsymbol{u}+\boldsymbol{u} \nabla & =0,
\end{aligned}
$$

In (2) $\theta$ and components $\left(u_{k}, \sigma_{i k}\right)$ are functions of $\left(x_{0}, t\right)$, and $\left(\nabla, \nabla^{2}, 1\right)$ respectively are gradient and Laplacian operators and identity tensor. Symbols $(D f, \dot{f})$ represent time differentiation in basis $x_{0}$ and

$$
\mathrm{P}=1+t_{0} D
$$

Here constants $(\mu, \rho, v)$ are shear modulus, mass density and Poisson's ratio, and $\left(k_{T}, C_{E}, \alpha_{D}\right)$ are thermal conductivity, specific heat at constant strain, and coefficient of (volumetric) thermal expansion. Constant $t_{0}$ is the thermal relaxation time. Equation (1) reflects assumptions that body forces can be ignored, and heat is neither added to, nor extracted from, the solid. Partial uncoupling of (2a) and (2b) gives

$$
u=u_{S}+u_{D}
$$




$$
\begin{aligned}
&\left(\nabla^{2}-D_{S}^{2}\right) \boldsymbol{u}_{S}=0, \nabla \cdot \boldsymbol{u}_{S}=0, \\
&\left(c_{D}^{2} \nabla^{2}-D_{S}^{2}\right) \boldsymbol{u}_{D}-\alpha_{D} \nabla \theta=0, \quad \nabla \times \boldsymbol{u}_{D}=0, \\
& {\left[\left(c_{D}^{2} \nabla^{2}-D_{S}^{2}\right)\left(h \nabla^{2}-D_{S} \mathrm{P}\right)-\varepsilon D_{S} \mathrm{P} \nabla^{2}\right]\left(\boldsymbol{u}_{D}, \theta\right)=0 . }
\end{aligned}
$$

In (4) $D=V_{S} D_{S}$, and a modification of (3) was used:

$$
\mathrm{P}=1+h_{0} D_{S}
$$

Equation (4) also introduces parameters

$$
\begin{gathered}
V_{S}=\sqrt{\frac{\mu}{\rho}}, \quad V_{D}=c_{D} V_{S}, \quad c_{D}=\sqrt{2 \frac{1-v}{1-2 v}}, \quad \varepsilon=\frac{\mu T_{0}}{\rho C_{E}} \alpha_{D}, \\
h=\frac{k_{T}}{C_{E} \sqrt{\mu \rho}}, \quad h_{0}=V_{S} t_{0} .
\end{gathered}
$$

In (6) $\varepsilon$ is the dimensionless thermal coupling constant, and $\left(h, h_{0}\right)$ are thermoelastic characteristic lengths. Symbols $\left(V_{S}, V_{D}\right)$ are, respectively, shear speed and isothermal dilatational speed. In light of restriction (1b), it is noted that $V_{R}<V_{S}$ and that $V_{R}$ will be shown to depend both on material properties and the nature of the point forces.

For $x_{3}^{0}=0 \pm,\left(x_{1}^{0}, x_{2}^{0}\right) \in A_{C}+\delta A(t>0)$ :

$$
\sigma_{3 k}=-P_{k} \delta\left(x_{1}^{0}\right) \delta\left(x_{2}^{0}\right), \quad \frac{\partial \theta}{\partial x_{3}^{0}}=0 .
$$

For $x_{3}^{0}=0,\left(x_{1}^{0}, x_{2}^{0}\right) \notin A_{C}+\delta A(t>0)$,

$$
\left[u_{k}\right]=[\theta]=0 .
$$

In (7) force $P_{k}$ is a positive constant, $\delta(f)$ denotes Dirac function, and $[f]=f^{(+)}-f^{(-)}$where $f^{( \pm)}=f\left(x_{1}^{0}, x_{2}^{0}, 0 \pm, t\right)$. Equation (7a) reflects the assumption that thermal convection on the crack faces is negligible. In addition $\left[u_{k}\right]$ and $[\theta]$ must vanish continuously on $C$, but $\sigma_{3 k}$ can exhibit (integrable) singular behavior on $C$. For $t \leq 0,(\boldsymbol{u}, \boldsymbol{T}, \theta) \equiv 0$ and for finite $t>0,(\boldsymbol{u}, \boldsymbol{T}, \theta)$ must be bounded as $\left|x_{0}\right| \rightarrow \infty$.

\section{Discontinuity problem}

A common practice for solving crack problems is to represent the relative motion of crack faces as unknown discontinuities in displacement, e.g., [Barber 1992]. To implement that procedure, the related problem of discontinuities in $\left(u_{k}, \sigma_{3 k}, \theta, \partial \theta / \partial x_{3}^{0}\right)$ is now considered: The unbounded solid is again at rest at uniform (absolute) temperature $T_{0}$ when for time $t>0$ the discontinuities are imposed in the same region $A_{C}+\delta A$ of the $x_{1}^{0} x_{2}^{0}-$ plane. In place of (7) we have for $x_{3}^{0}=0,\left(x_{1}^{0}, x_{2}^{0}\right) \in A_{C}+\delta A(t>0)$ :

$$
\left[u_{k}\right]=\Delta_{k}, \quad\left[\sigma_{3 k}\right]=\Sigma_{k}, \quad[\theta]=\Theta, \quad\left[\partial \theta / \partial x_{3}^{0}\right]=\mathrm{d} \Theta
$$

For $x_{3}^{0}=0,\left(x_{1}^{0}, x_{2}^{0}\right) \notin A_{C}+\delta A(t>0)$,

$$
\left[u_{k}\right]=\left[\sigma_{3 k}\right]=[\theta]=\left[\partial \theta / \partial x_{3}^{0}\right]=0 .
$$


Here $\left(\Delta_{k}, \Sigma_{k}, \Theta, \mathrm{d} \Theta\right)$ are continuous functions of $\left(x_{1}^{0}, x_{2}^{0}, t\right)$. They vanish on $C$ and for $t \leq 0$ are bounded in $A_{C}+\delta A$ for $\sqrt{\left(x_{1}^{0}\right)^{2}+\left(x_{2}^{0}\right)^{2}} \rightarrow \infty$. Therefore, as in the crack problem, $(\boldsymbol{u}, \boldsymbol{T}, \theta) \equiv 0$ for $t \leq 0$ and are bounded as $\left|x_{0}\right|$ for finite $t>0$.

\section{Transform solution}

An effective procedure, e.g., [Brock and Achenbach 1973], for 2D transient study of semi-infinite crack extension at constant speed employs

(a) coordinates that translate with the crack edge, and

(b) unilateral temporal and bilateral spatial integral transform [Sneddon 1972].

In view of (1) a translating basis $x$ is defined for $|\psi|<\frac{1}{2} \pi$ as

$$
\begin{gathered}
x_{1}=x_{1}^{0}-[c(\psi) \cos \psi] s, \quad x_{2}=x_{2}^{0}-[c(\psi) \sin \psi] s, \quad x_{3}=x_{3}^{0}, \\
s=V_{S} t, \quad c(\psi)=\frac{V(\psi)}{V_{S}}, \\
D f=\dot{f}=V_{S}\left[\partial_{S} f-c(\psi)\left(\partial_{1} f \cos \psi+\partial_{2} f \sin \psi\right)\right], \\
\partial_{S} f=\frac{\partial f}{\partial s}, \quad \partial_{k} f=\frac{\partial f}{\partial x_{k}}, \quad k=(1,2,3) .
\end{gathered}
$$

The temporal Laplace transform operation is

$$
L(f)=\hat{f}=\int f(s) \exp (-p s) \mathrm{d} s
$$

Integration is over positive real $s$ and $\operatorname{Re}(p)>0$. A double spatial integral transform and inversion, respectively, can be defined [Sneddon 1972] by

$$
\begin{aligned}
& \tilde{f}\left(p, q_{1}, q_{2}\right)=\iint \hat{f}\left(p, x_{1}, x_{2}\right) \exp \left[-p\left(q_{1} x_{1}+q_{2} x_{2}\right)\right] \mathrm{d} x_{1} \mathrm{~d} x_{2}, \\
& \hat{f}\left(p, x_{1}, x_{2}\right)=\left(\frac{p}{2 \pi i}\right)^{2} \iint \tilde{f}\left(p, q_{1}, q_{2}\right) \exp \left[p\left(q_{1} x_{1}+q_{2} x_{2}\right)\right] \mathrm{d} q_{1} \mathrm{~d} q_{2} .
\end{aligned}
$$

Integration in (10b) is over real $\left(x_{1}, x_{2}\right)$; integration in (10c) is along the imaginary $\left(q_{1}, q_{2}\right)$-axes. It is noted that $(\boldsymbol{x}, s)$ have dimensions of length, $p$ has dimensions of inverse length, and $\left(q_{1}, q_{2}\right)$ are dimensionless. Because (1) involves a speed that varies with direction, application of (9) and (10b) to (2)-(5) and (7) is complicated. Despite use of $\psi$ the discontinuity problem is not axially symmetric. However, 3D studies of sliding and rolling contact [Brock 2012] and crack growth [Brock 2017a; 2017b] suggest transformations

$$
\begin{aligned}
\operatorname{Im}\left(q_{1}\right) & =\operatorname{Im}(q) \cos \psi, & \operatorname{Im}\left(q_{2}\right) & =\operatorname{Im}(q) \sin \psi, \\
x_{1} & =x \cos \psi, & x_{2} & =x \sin \psi .
\end{aligned}
$$

Here $\operatorname{Re}(q)=0+,|\operatorname{Im}(q)|,|x|<\infty$ and $|\psi|<\frac{1}{2} \pi$. Parameters $(x, \psi)$ and $(q, \psi)$ resemble quasipolar coordinates, i.e.,

$$
\mathrm{d} x_{1} \mathrm{~d} x_{2}=|x| \mathrm{d} x \mathrm{~d} \psi, \quad \mathrm{d} q_{1} \mathrm{~d} q_{2}=|q| \mathrm{d} q \mathrm{~d} \psi .
$$


The uncoupling effect of (11) leads to the combination

$$
\begin{aligned}
\tilde{f}\left(p, q_{1}, q_{2}\right) & \rightarrow \bar{f}(p, q, \psi), \\
\hat{f}(p, x, \psi) & =-\frac{p^{2}}{2 \pi} \int \frac{|q|}{q} \bar{f}(p, q, \psi) \exp (p q x) \mathrm{d} q .
\end{aligned}
$$

Integration is along the positive $(\operatorname{Re}(q)=0+)$ side of the $\operatorname{Im}(q)$-axis.

In view of (9)-(11) and (12a), (4) and (5) give a corresponding set in transform space by making formal substitutions

$$
\begin{gathered}
\nabla \rightarrow\left(p q \cos \psi, p q \sin \psi, \partial_{3}\right), \quad D_{S} \rightarrow p \beta, \quad \nabla^{2} \rightarrow \partial_{3}^{2}+p^{2} q^{2}, \\
\mathrm{P} \rightarrow 1+h_{0} p \beta, \\
\beta=1-c q .
\end{gathered}
$$

Set elements that correspond to (4b)-(4d) are homogeneous, ordinary differential equations in $x_{3}$, with characteristic functions $p B(q)$ and $A_{ \pm}(p, q)$ :

$$
\begin{aligned}
B(q) & =\sqrt{\beta^{2}-q^{2}}, \\
A_{ \pm}(p, q) & =p \sqrt{\left(\frac{2 \beta}{\Gamma_{+} \pm \Gamma_{-}}\right)^{2}-q^{2}} \\
\Gamma_{ \pm} & =\sqrt{\left[\sqrt{\frac{h p \beta}{1+h_{0} p \beta} \pm c_{D}}\right]^{2}+\varepsilon}
\end{aligned}
$$

Focus of this transient study is on the initiation phase of fracture, i.e., small $t$ (and therefore small $s$ ). The Lord and Shulman [1967] model is quite robust for this purpose. Indeed [Brock 2009; Ignaczak and Ostoja-Starzewski 2010] indicate that $h \approx O\left(10^{-9}\right) \mathrm{m}, h_{0} \approx O\left(10^{-10}\right) \mathrm{m}$ so that, in view of (10a), transform expressions valid for $\left|h_{0} p\right| \gg 1$ are sufficient. Result (14b) assumes the form $p A_{ \pm}(q)$ defined below, and the set corresponding to (4b)-(4d) gives general solutions:

$$
\begin{gathered}
\overline{\boldsymbol{u}}_{S}\left[U_{1}^{( \pm)}, U_{2}^{( \pm)},( \pm) \frac{q}{B}\left(U_{1}^{( \pm)} \cos \psi+U_{2}^{( \pm)}\right)\right] \exp \left(-p B\left|x_{3}\right|\right) \\
\overline{\boldsymbol{u}}_{D}=\overline{\boldsymbol{u}}_{+}+\overline{\boldsymbol{u}}_{--}, \quad \bar{\theta}=\bar{\theta}_{+}+\bar{\theta}_{-} \\
\overline{\boldsymbol{u}}_{ \pm}=\left[q \cos \psi, q \sin \psi,(\mp) A_{ \pm}\right] U_{ \pm}^{( \pm)} \exp \left(-p A_{ \pm}\left|x_{3}\right|\right) \\
\bar{\theta}_{ \pm}=-C_{ \pm} \frac{\beta^{2}}{\alpha_{D}} p U_{ \pm}^{( \pm)} \exp \left(-p A_{ \pm}\left|x_{3}\right|\right)
\end{gathered}
$$

Here $\left(U_{ \pm}^{( \pm)}, U_{1}^{( \pm)}, U_{2}^{( \pm)}\right)$are unknown functions of $(p, q, \psi)$ and $( \pm)$ signifies $x_{3}>0(+), x_{3}<0(-)$. In addition,

$$
\begin{gathered}
A_{ \pm}=\sqrt{\beta^{2} / c_{ \pm}^{2}-q^{2}}, \\
C_{ \pm}=1-c_{D}^{2} / c_{ \pm}^{2}, \quad c_{ \pm}=\frac{1}{2}\left(\Gamma_{+} \pm \Gamma_{-}\right), \\
\Gamma_{ \pm}=\sqrt{\left(\sqrt{\lambda} \pm c_{D}\right)^{2}+\varepsilon}, \quad \lambda=h / h_{0} .
\end{gathered}
$$


Combinations of terms in (16) also prove useful:

$$
\begin{aligned}
C_{+}-C_{-} & =\Omega=\frac{\Gamma_{+} \Gamma_{-}}{\lambda}, & C_{+} C_{-} & =-\frac{\varepsilon}{\lambda}, \\
c_{+}^{2}-c_{-}^{2} & =\Gamma_{+} \Gamma_{-}, & c_{+} c_{-} & =c_{D} \sqrt{\lambda} .
\end{aligned}
$$

The dimensionless terms $c_{ \pm}$in (16) show that solution behavior is governed by the three wave speeds $\left(V_{S}, V_{ \pm}=c_{ \pm} V_{S}\right)$ where $1<c_{-}<c_{+}$. Data, e.g., [Brock 2009; Ignaczak and Ostoja-Starzewski 2010], suggest moreover that $c_{+}>c_{D}, c_{-} \approx c_{D}$ - so that $V_{+}$is larger than isothermal dilatational wave speed $V_{D}=c_{D} V_{S}$ while $V_{-}$is approximately the same. Bounded behavior for $\left(\hat{u}_{k}, \hat{\theta}\right)$ as $\left|x_{3}\right| \rightarrow \infty$ requires in light of (15) that $\operatorname{Re}\left(A_{ \pm}\right)>0$ and $\operatorname{Re}(B)>0$ in the $q$-plane with, respectively, branch cuts

$$
\begin{aligned}
& \operatorname{Im}(q)=0, \quad \frac{-1}{c_{ \pm}-c}<\operatorname{Re}(q)<\frac{1}{c_{ \pm}+c} \\
& \operatorname{Im}(q)=0, \quad \frac{-1}{1-c}<\operatorname{Re}(q)<\frac{1}{1+c}
\end{aligned}
$$

It is noted that (18) is valid only so long as $c<1$; i.e., $V(\psi)<V_{S}\left(|\psi|<\frac{1}{2} \pi\right)$.

\section{Application to fracture problem: equations for solution}

In order that (15) and results in Appendix A represent the transform solution for the fracture problem, the transforms of (7) must be satisfied. Because (7) does not involve $\left(\Sigma_{k}, \mathrm{~d} \Theta\right)$, their transforms can be dropped. Use of (2c), (9), (10a), (10b), (11) and (12b) then give five equations for transforms of $\left(\sigma_{3 k}, \theta^{( \pm)}, \partial_{3} \theta\right)$ along $x_{3}=0$ in terms of transforms of $\left(\Delta_{k}, \Theta\right)$. It is noted that for $\left(s>0, x_{3}=0\right)$,

$$
\begin{aligned}
& \sigma_{3 k}=\sigma_{3 k}^{C}-P_{k} \delta\left(x_{1}^{0}\right) \delta\left(x_{2}^{0}\right), \\
& \partial_{3} \theta=\partial_{3} \theta^{C} .
\end{aligned}
$$

Here $\left(\sigma_{3 k}^{C}, \partial_{3} \theta^{C}\right)$ exists for $x>0$ in a region generated behind wave front $c_{+} s-x-c s>0$. Thus the corresponding transform exists for $\operatorname{Re}(q)>-1 /\left(c_{+}-c\right)$. The Dirac function term has transform

$$
-\frac{P_{k}}{p \beta}(\operatorname{Re}(q)<1 / c)
$$

Function $\left(\Delta_{k}, \Theta\right)$ occurs for $x<0$ in a region generated behind wave front $c_{+} s+x+c s>0$. Thus the corresponding transform exists for $\operatorname{Re}(q)<1 /\left(c_{+}+c\right)$. Four transform equations can be written and are given in Appendix B. There it is noted that

$$
\begin{array}{rlrl}
M_{12}\left(q_{12}\right) & =0, & M_{12} \approx R_{12} q^{4}(|q| \rightarrow \infty), & q_{12}=\frac{ \pm 1}{c_{12} \pm c} \\
M_{3}\left(q_{3}\right)=0, & M_{3} \approx R_{3} q^{4}(|q| \rightarrow \infty), & q_{3}=\frac{ \pm 1}{c_{3} \pm c} \\
M_{ \pm}\left(q_{R}^{ \pm}\right)=0, & M_{ \pm} q^{4}(|q| \rightarrow \infty), & q_{R}^{ \pm}=\frac{1}{c_{R}^{ \pm}+c}, \frac{-1}{c_{R}^{ \pm}-c} .
\end{array}
$$


In $(21),\left(R_{12}, R_{3}, R_{ \pm}\right)$are functions of $c$ :

$$
\begin{array}{lll}
R_{12}=C_{+} R_{-}-C_{-} R_{+}, & R_{12}\left(c_{12}^{2}\right)=0 & \left(0<c_{12}<1\right), \\
R_{3}=C_{+} a_{+} \frac{R_{-}}{b}-C_{-} a_{-} \frac{R_{+}}{b}, & R_{3}\left(c_{3}^{2}\right)=0 & \left(0<c_{3}<1\right), \\
R_{ \pm}=4 a_{ \pm} b-k^{2}, & R_{ \pm}(0)=R_{ \pm}\left(\left(c_{R}^{ \pm}\right)^{2}\right)=0 \quad\left(0<c_{R}^{ \pm}<1\right), \\
a_{ \pm}(c)=\frac{1}{c_{ \pm}} \sqrt{c_{ \pm}^{2}-c^{2}}, & b(c)=\sqrt{1-c^{2}}, & k(c)=c^{2}-2 .
\end{array}
$$

Terms $\left(R_{ \pm}, M_{ \pm}\right)$have the form of the Rayleigh function [Achenbach 1976]. Thus $\left(R_{12}, R_{3}\right)$ and $\left(M_{12}, M_{3}\right)$ are thermoelastic Rayleigh functions of respectively, variables $c$ and $q$. Data, e.g., [Brock 2009; Ignaczak and Ostoja-Starzewski 2010] indicate that in general,

$$
0<c_{3}<c_{12}<1<c_{-}<c_{+}, \quad c_{-} \approx c_{D}-
$$

In view of (6) and (9) the fracture problem solution is governed by both $\left(V_{S}, V_{ \pm}=c_{ \pm} V_{S}\right)$ and Rayleigh speeds $V_{12}=c_{12} V_{S}$ and $V_{3}=c_{3} V_{S}$. Moreover, subcritical speed is defined as $V(\psi)<V_{3}\left(|\psi|<\frac{1}{2} \pi\right)$.

\section{Solution: Wiener-Hopf equation}

The four equations, (B.1) and (B.2), involve eight unknown transforms $\left(\bar{\sigma}_{3 k}^{C}, \partial_{3} \bar{\theta}^{C}\right)$ and $\left(\bar{\Delta}_{k}, \bar{\Theta}\right)$. The regions of analyticity in the $q$-plane of the two transform sets differ but can also overlap. Thus (B.1) and (B.2) can be viewed as a set of coupled equations of the Wiener-Hopf type [Morse and Feshbach 1953; Achenbach 1976].

Equation (B.1c). It is noted that $\left(A_{ \pm}, B\right)$ form products $\left(A_{+}^{+} A_{+}^{-}, A_{-}^{+} A_{-}^{-}, B^{+} B^{-}\right)$where

$$
\begin{gathered}
A_{+}^{ \pm}=\frac{1}{\sqrt{c_{+}}} \sqrt{1 \pm q\left(c_{+} \mp c\right)}, \quad A_{-}^{ \pm}=\frac{1}{\sqrt{c_{-}}} \sqrt{1 \pm q\left(c_{-} \mp c\right)}, \\
B^{\mp}=\sqrt{1 \pm q(1 \mp c)} .
\end{gathered}
$$

In (24a) $\left(A_{ \pm}^{+}, A_{ \pm}^{-}\right)$are analytic in, respectively, overlapping half-planes $\operatorname{Re}(q)>-1 /\left(c_{ \pm}-c\right)$ and $\operatorname{Re}(q)<$ $1 /\left(c_{ \pm}+c\right)$. Terms $\left(B^{+}, B^{-}\right)$in $(24 b)$ are analytic in overlapping half-planes $\operatorname{Re}(q)>-1 /(1-c)$ and $\operatorname{Re}(q)<1 /(1+c)$. Study of $M_{3}$ in (B.3b) leads to construction of function

$$
G_{3}=-\frac{M_{3}}{\beta^{2}} \frac{c^{2}}{R_{3}} \frac{c_{3}^{2}-c^{2}}{\left[1+q\left(c_{3}-c\right)\right]\left[1-q\left(c_{3}+c\right)\right]} .
$$

Here $G_{3} \rightarrow 1(|q| \rightarrow \infty)$, and has no zeros in the $q$-plane with branch cut $\operatorname{Im}(q)=0,-1 /(1-c)<\operatorname{Re}(q)<$ $-1 /\left(c_{+}-c\right), 1 /\left(c_{+}+c\right)<\operatorname{Re}(q)<1 /(1+c)$. Therefore $G_{3}$ forms $G_{3}^{+} G_{3}^{-}$, where $\left(G_{3}^{+}, G_{3}^{-}\right)$are defined by (C.1a) and are analytic in overlapping half-planes $\operatorname{Re}(q)>-1 /\left(c_{+}-c\right)$ and $\operatorname{Re}(q)<1 /\left(c_{+}+c\right)$. Equation (B.1c) can then be written as

$$
\begin{array}{r}
\frac{F^{+}}{G_{3}^{+}} \bar{\sigma}_{33}^{C} \frac{c_{3}-c}{1+q\left(c_{3}-c\right)}-\frac{P_{3}}{p \beta}\left[\frac{F^{+}}{G_{3}^{+}} \frac{c_{3}-c}{1+q\left(c_{3}-c\right)}-\frac{\sqrt{c}}{g_{3}^{+}}\left(1-\frac{c}{c_{3}}\right)\right] \\
=-\mu \frac{\Omega R_{3}}{2 c^{2}} \frac{G_{3}^{-}}{F^{-}}\left(\frac{1}{c_{3}+c}-1\right) p \bar{\Delta}_{3}+\frac{p_{3}}{p \beta} \frac{\sqrt{c}}{g_{3}^{+}}\left(1-\frac{c}{c_{3}}\right),
\end{array}
$$




$$
\begin{gathered}
F^{+}=\frac{A_{+}^{+} A_{-}^{+}}{B^{+}}, \quad F^{-}=\frac{A_{+}^{-} A_{-}^{-}}{B^{-}}, \\
A_{ \pm}^{+}\left(\frac{1}{c}\right)=B^{+}\left(\frac{1}{c}\right)=F^{+}\left(\frac{1}{c}\right)=\frac{1}{\sqrt{c}}, \quad g_{3}^{+}=G_{3}^{+}\left(\frac{1}{c}\right) .
\end{gathered}
$$

The left-hand and right-hand sides of (26a) are analytic for respectively the overlapping half-planes $\operatorname{Re}(q)>-1 /\left(c_{+}-c\right)$ and $\operatorname{Re}(q)<1 /\left(c_{+}+c\right)$ so that each side is an analytic continuation of the same entire function. In connection with (7), $\Delta_{k}$ must vanish continuously on $C$ for $x \rightarrow 0$-. Equation (10a) and (12b) therefore require that $p q \bar{\Delta}_{k}$, and also the right-hand side of (26a), vanish for $|q| \rightarrow \infty$. The entire function itself must then in light of Liouville's theorem [Morse and Feshbach 1953] vanish, and (26a) leads to

$$
\begin{aligned}
\bar{\Delta}_{3} & =\frac{2 \sqrt{c} \beta}{\mu p^{2} g_{3}^{+}} \frac{F^{-} G_{3}^{+}}{c_{3} \Omega M_{3}}\left[1+q\left(c_{3}-c\right)\right] P_{3}, \\
\bar{\sigma}_{33}^{C} & =-\frac{\sqrt{c} P_{3}}{c_{3} p g_{3}^{+}} \frac{G_{3}^{+}}{\beta F^{+}}\left[1+q\left(c_{3}-c\right)\right] .
\end{aligned}
$$

Examination of the fracture problem solution requires knowledge of $\left(\bar{\sigma}_{33}^{C}, \dot{\Delta}_{3}\right)$ for $x \rightarrow 0-$ and $x \rightarrow 0+$, respectively. In view of (9)-(11),

$$
\dot{f}=V_{S}\left(\partial_{S}-c \partial\right) f, \quad \partial f=\frac{\partial f}{\partial x} .
$$

Therefore (13a) shows that expressions for transforms $\left(\bar{\sigma}_{33}^{C}, p \beta \bar{\Delta}_{3}\right)$ that are valid for $|q| \rightarrow \infty$ suffice in this regard, and are given by (D.2c) and (D.5c).

Equation (B.1a) and (B.1b). Algebraic manipulation of (B.1a) and (B.1b) leads to a partial uncoupling:

$$
\begin{aligned}
& \left(\bar{\sigma}_{31}^{C}-\frac{P_{1}}{p \beta}\right) \cos \psi+\left(\bar{\sigma}_{32}^{C}-\frac{P_{2}}{p \beta}\right) \sin \psi=\frac{\mu}{\Omega \beta^{2}}\left(\frac{M_{12}}{2 B} p \bar{\Delta}_{P}+q M_{A} \alpha_{D} \bar{\Theta}\right), \\
& \left(\bar{\sigma}_{31}^{C}-\frac{P_{1}}{p \beta}\right) \sin \psi-\left(\bar{\sigma}_{32}^{C}-\frac{P_{2}}{p \beta}\right) \cos \psi=\mu B p \bar{\Delta}_{M} .
\end{aligned}
$$

In view of (24b), (29b) can written in Wiener-Hopf form (compare (26a)):

$$
\begin{aligned}
\frac{2}{B^{+}} \bar{\sigma}_{M}-\frac{2}{p \beta}\left(\frac{1}{B^{+}}-\sqrt{c}\right)\left(P_{1} \sin \psi-P_{2} \cos \psi\right) & =\mu p B^{-} \bar{\Delta}_{M}+\frac{2 \sqrt{c}}{p \beta}\left(P_{1} \sin \psi-P_{2} \cos \psi\right), \\
\bar{\sigma}_{M} & =\bar{\sigma}_{31}^{C} \sin \psi-\bar{\sigma}_{32}^{C} \cos \psi
\end{aligned}
$$

Behavior of $\Delta_{k}$ for $x \rightarrow 0-$ dictates that both sides of (30a) vanish. Therefore,

$$
\begin{aligned}
& \bar{\sigma}_{M}=\frac{1}{p \beta}\left(1-\sqrt{c} B^{+}\right)\left(P_{1} \sin \psi-P_{2} \cos \psi\right), \\
& \bar{\Delta}_{M}=\frac{-2 \sqrt{c}}{\mu p^{2} \beta B^{-}}\left(P_{1} \sin \psi-P_{2} \cos \psi\right) .
\end{aligned}
$$


Equation (B.2) and (29a). Equations (29a) and (B.2) are treated as linear equations for $\left(\bar{\Theta}, \bar{\Delta}_{P}\right)$, and are solved simultaneously to yield

$$
\begin{aligned}
\alpha_{D} \bar{\Theta} & =\frac{M_{12}}{B M_{3}}\left(\frac{2}{p} \alpha_{D} \partial_{3} \bar{\theta}^{C}\right)+4 q \frac{M_{A}}{M_{3}} \frac{\varepsilon}{\lambda} \frac{\beta^{2}}{\mu}\left[\bar{\sigma}_{P}-\left(P_{1} \cos \psi+P_{2} \sin \psi\right)\right], \\
p \bar{\Delta}_{P} & =-4 q \frac{M_{A}}{M_{3}}\left(\frac{2}{p} \alpha_{D} \partial_{3} \bar{\theta}^{C}\right)+\frac{2 M_{C}}{M_{3}} \frac{\beta^{2}}{\mu}\left[\bar{\sigma}_{P}-\left(P_{1} \cos \psi+P_{2} \sin \psi\right)\right], \\
\bar{\sigma}_{P} & =\bar{\sigma}_{31}^{C} \cos \psi+\bar{\sigma}_{32}^{C} \sin \psi .
\end{aligned}
$$

As in the case of $M_{3}$ functions related to $\left(M_{12}, M_{A}, M_{C}\right)$ can be defined as

$$
\begin{aligned}
G_{12} & =-\frac{M_{12}}{\beta^{2}} \frac{c^{2}}{R_{12}} \frac{c_{12}^{2}-c^{2}}{\left[1+q\left(c_{12}-c\right)\right]\left[1-q\left(c_{12}+c\right)\right]}=G_{12}^{+} G_{12}^{-}, \\
G_{A} & =\frac{M_{A} a_{+}(c)}{A_{+} m_{A}(c)}=G_{A}^{+} G_{A}^{-}, \quad m_{A}(c)=a_{+}(c)-a_{-}(c), \\
G_{C} & =\frac{M_{C} a_{+}(c)}{A_{+} m_{C}(c)}=G_{C}^{+} G_{C}^{-}, \quad m_{C}(c)=C_{+} a_{+}(c)-C_{-} a_{-}(c) .
\end{aligned}
$$

Functions $\left(G_{12}^{+}, G_{A}^{+}, G_{C}^{+}\right)$and $\left(G_{12}^{-}, G_{A}^{-}, G_{C}^{-}\right)$are analytic in overlapping halves of the cut $q$-plane, and are given by (C.2) and (C.4), respectively. Factorization based on these results does not in general put (32a) and (32b) in standard Wiener-Hopf form [Achenbach 1976]. Equation (24a) and (24b) show for $|q| \rightarrow \infty$ however that

$$
\begin{array}{ll}
A_{ \pm}^{+} \approx \sqrt{q} \sqrt{1+c / c_{ \pm}}, & B^{+} \approx \sqrt{q} \sqrt{1+c} \quad(\operatorname{Re}(q)>0-), \\
A_{ \pm}^{-} \approx \sqrt{-q} \sqrt{1-c / c_{ \pm}}, & B^{-} \approx \sqrt{-q} \sqrt{1-c} \quad(\operatorname{Re}(q)<0+) .
\end{array}
$$

Equations (21), (B.3) and (34) show that (32) for $|q| \rightarrow \infty$ depends on $(\beta, \sqrt{ \pm q})$, and that $\sqrt{ \pm q}$ define overlapping half-planes $\operatorname{Re}(q)>0-$ and $\operatorname{Re}(q)<0+$ as regions of analyticity. As exemplified by (D.1), (D.2c) and (D.5c), functions of $\sqrt{ \pm q}$ are sufficient for study of solution behavior when $|x| \approx$ 0 . Equation (32a) and (32b) then yield Wiener-Hopf equations whose solutions are combined with asymptotic forms for (31). This process leads to (D.2a) and (D.2b) valid for $x \rightarrow 0+$, and (D.4) and (D.5) valid for $x \rightarrow 0$-.

\section{Transform inversions valid on crack plane near $C$}

For $(1 / \sqrt{q}, \sqrt{q})$ and $1 / \sqrt{-q}$ respectively inverse operation (12b) yields

$$
\begin{gathered}
-\frac{p^{2}}{\pi \sqrt{x}} \int_{+} \frac{\mathrm{d} u}{\sqrt{u}} \exp (-p u), \quad \frac{p}{2 \pi} \frac{1}{x^{3 / 2}} \int_{+} \frac{\mathrm{d} u}{\sqrt{u}} \exp (-p u) \quad(x>0), \\
-\frac{p^{2}}{\pi \sqrt{-x}} \int_{+} \frac{\mathrm{d} u}{\sqrt{u}} \exp (-p u) \quad(x<0) .
\end{gathered}
$$

The "+" signifies integration over the entire positive real $u$-axis. In view of (37) functions in Appendix D involve $p \exp (-p u)$, and its inverse is recognized as $\partial_{S} \delta(s-u)$ [Abramowitz and Stegun 1972]. The 
point force represents a step-function in time, so for generality we now consider the case

$$
P_{k}=P_{k}\left(V_{S} t\right), \quad P_{k}(0)=0 .
$$

Clarity of solution is enhanced if points in the $x_{1}^{0} x_{2}^{0}$-plane are located with respect to fixed point $x_{0}=0$. Therefore the inverses from (D.1)-(D.3) are, by convolution, written as functions of $\left(x_{0}, \psi, s\right)$, where $x_{0}=x+c s$, and for $\left(s>0, x_{0} \rightarrow c s+,|\psi|<\frac{1}{2} \pi\right)$,

$$
\begin{gathered}
\alpha_{D} \partial_{3} \theta^{C} \approx-\frac{2 \varepsilon}{\mu \Gamma_{+} \Gamma_{-}} \frac{m_{A} m_{C} K_{I I}}{\sqrt{c}\left(x_{0}-c s\right)^{3 / 2}}, \\
\sigma_{31}^{C} \approx \frac{1}{\sqrt{c} \sqrt{x_{0}-c s}}\left[\sin \psi \sqrt{1-c} K_{I I I}+\cos \psi \frac{R_{3}}{c^{2}} N_{12} K_{I I}\right], \\
\sigma_{32}^{C} \approx \frac{1}{\sqrt{c} \sqrt{x_{0}-c s}}\left[-\cos \psi \sqrt{1-c} K_{I I I}+\sin \psi \frac{R_{3}}{c^{2}} N_{12} K_{I I}\right], \\
\sigma_{33}^{C} \approx \frac{K_{1}}{\sqrt{c} \sqrt{x_{0}-c s}} \frac{c^{2}}{R_{3}} \frac{\sqrt{c+c-} \sqrt{1-c}}{\sqrt{c_{+}-c} \sqrt{c_{-}-c}} .
\end{gathered}
$$

In similar fashion (D.4) and (D.5) yield for $\left(s>0, x_{0} \rightarrow c s-,|\psi|<\frac{1}{2} \pi\right)$,

$$
\begin{aligned}
\alpha_{D} \Theta & \approx \frac{4 m_{A}}{\mu \sqrt{c s-x_{0}}} \frac{\varepsilon}{\lambda} K_{I I}, \\
\dot{\Delta}_{1} & \approx \frac{2 V_{S} \sqrt{c}}{\mu \sqrt{c s-x_{0}}}\left[\frac{\sin \psi}{\sqrt{1+c}} K_{I I I}+m_{C} \cos \psi K_{I I}\right], \\
\dot{\Delta}_{2} & \approx \frac{2 V_{S} \sqrt{c}}{\mu \sqrt{c s-x_{0}}}\left[\frac{\cos \psi}{\sqrt{1+c}} K_{I I I}+m_{C} \sin \psi K_{I I}\right], \\
\dot{\Delta}_{3} & \approx \frac{2 V_{S} \sqrt{c} \Omega}{\mu \sqrt{c s-x_{0}}} \frac{\sqrt{c_{+}+c} \sqrt{c_{-}+c}}{\sqrt{c_{+}-c_{-}} \sqrt{1+c}} K_{I} .
\end{aligned}
$$

In (37)-(40),

$$
\begin{aligned}
K_{I} & =\frac{c^{2}}{\pi g_{3}^{+} R_{3}}\left(1-\frac{c}{c_{3}}\right) \partial_{S} \int_{0}^{s} \frac{\mathrm{d} u}{\sqrt{s-u}} \frac{\mathrm{d}}{\mathrm{d} u} P_{3} \\
K_{I I} & =\frac{c^{2}}{\pi g_{3}^{+} R_{3}}\left(1-\frac{c}{c_{3}}\right) \partial_{S} \int_{0}^{s} \frac{\mathrm{d} u}{\sqrt{s-u}} \frac{\mathrm{d}}{\mathrm{d} u}\left(P_{1} \cos \psi+P_{2} \sin \psi\right) \\
K_{I I I} & =\frac{1}{\pi} \partial_{S} \int_{0}^{s} \frac{\mathrm{d} u}{\sqrt{s-u}} \frac{\mathrm{d}}{\mathrm{d} u}\left(P_{1} \sin \psi+P_{2} \cos \psi\right)
\end{aligned}
$$

The roman numeral subscripts reflect the observation that, in a 2D study ( $\psi=0)$ terms (41a), (41b) and (41c) would be associated with, respectively, the opening, in-plane shear and antiplane shear modes of fracture [Freund 1990]. 


\section{Transform inversions valid near $C$}

Expressions for $\left(\dot{u}_{k}, \theta\right)$ near $C$ for $\left(x_{3}^{0}, x_{3}\right) \neq 0$ are also required. In view of (9c) and (28), $L\left(\dot{u}_{k}\right)=\beta p \bar{u}_{k}$ and $\left(\bar{u}_{k}, \bar{\theta}\right)$ can be generated from (15) and Appendix A by setting $\left(\mathrm{d} \bar{\Theta}, \bar{\Sigma}_{k}\right)=0$ and substituting results from Appendix D. For $x_{3} \neq 0$, a more explicit version of inversion formula (12) is useful:

$$
\begin{gathered}
\tilde{f}\left(p, q_{1}, q_{2}, x_{3}\right) \rightarrow f_{\Psi}(p, q, \psi) \exp \left(-p A\left|x_{3}\right|\right), \quad A=\left(A_{ \pm}, B\right), \\
\hat{f}\left(p, x, \psi, x_{3}\right)=-\frac{p^{2}}{2 \pi} \int \frac{|q|}{q} f_{\Psi}(p, q, \psi) \exp \left[p\left(q x-A\left|x_{3}\right|\right)\right] \mathrm{d} q .
\end{gathered}
$$

Result (37) follows from use of Cauchy theory to change the integration path in (12b) to the $\operatorname{Re}(q)$-axis. For $(42 \mathrm{~b})$ the path is changed to a contour $q(A)$ in the complex $q$-plane along which the exponential term assumes the form $\exp (-p u)$, where $u$ is real and positive. Because inversions valid near $C$ are sufficient, local coordinates $(r, \psi, \phi)$ centered on the portion of $C$ that borders $\delta A$ are introduced:

$$
r=\sqrt{x^{2}+x_{3}^{2}}, \quad \phi=\tan ^{-1} \frac{x_{3}}{x}(|\phi|<\pi)
$$

In (42b) $q\left(A_{ \pm}\right)$and $q(B)$ for $r \approx 0$ have, respectively, asymptotic forms

$$
\begin{array}{ll}
-\frac{u}{4 Q_{ \pm}}, & Q_{ \pm}=\cos \phi \mp i a_{ \pm} \sin \phi, \\
-\frac{u}{r Q_{B}}, & Q_{B}=\cos \phi \mp i b \sin \phi .
\end{array}
$$

It is noted that (D.4) and (D.5), which depend on $1 / \sqrt{-q}$, are associated in Appendix with operator ( \pm ). In the case of contour $q(B)$ therefore, (35a) and (35b) respectively are replaced by

$$
-\frac{p}{\pi c} \frac{\mathrm{B}^{(+)}}{\sqrt{2 r}} \int_{+} \frac{\mathrm{d} u}{\sqrt{u}} \exp (-p u), \quad \text { (干) } \frac{p}{\pi c} \frac{\mathrm{B}^{(-)}}{\sqrt{2 r}} \int_{+} \frac{\mathrm{d} u}{\sqrt{u}} \exp (-p u) .
$$

In the case of contour $q\left(A_{ \pm}\right)$replacements are

$$
-\frac{p}{\pi c} \frac{\mathrm{A}_{ \pm}^{(+)}}{\sqrt{2 r}} \int_{+} \frac{\mathrm{d} u}{\sqrt{u}} \exp (-p u), \quad \text { (干) } \frac{p}{\pi c} \frac{\mathrm{A}_{ \pm}^{(-)}}{\sqrt{2 r}} \int_{+} \frac{\mathrm{d} u}{\sqrt{u}} \exp (-p u) .
$$

In (45), $\left(B^{( \pm)}, A_{ \pm}^{( \pm)}\right)$are factors of the real $(+)$and imaginary $(-)$parts of $\left(\sqrt{Q_{1}}, \sqrt{Q_{ \pm}}\right)$:

$$
\begin{array}{ll}
\mathrm{B}^{( \pm)}=\sqrt{1( \pm)(\cos \phi) / B_{\Phi}}, & B_{\Phi}=\sqrt{1-c^{2} \sin ^{2} \phi}, \\
\mathrm{A}_{ \pm}^{( \pm)}=\sqrt{1( \pm)(\cos \phi) / A_{\Phi}^{ \pm}}, & A_{\Phi}^{ \pm}=\sqrt{1-c^{2} / c_{ \pm}^{2} \sin ^{2} \phi} .
\end{array}
$$

Convolution involving (38) is again introduced, and results for $\dot{u}_{k}(r \rightarrow 0)$ follow:

$$
\begin{aligned}
\dot{u}_{1} \approx \frac{\sqrt{2 c}}{\mu \sqrt{r}}\left[\frac{\sqrt{c_{+}+c} \sqrt{c_{-}+c}}{c^{2} \sqrt{1+c} \sqrt{c_{+} c_{-}}} \mathrm{P}_{3}^{(+)} K_{I}-\frac{\mathrm{P}_{12}^{(-)}}{c^{2}} K_{I I} \operatorname{sgn}(\phi)\right] \cos \psi \\
-\frac{\sqrt{c}}{\mu \sqrt{2 r}} \frac{\mathrm{B}^{(-)} K_{I I I}}{\sqrt{1+c}} \operatorname{sgn}(\phi) \sin \psi,
\end{aligned}
$$




$$
\begin{aligned}
& \dot{u}_{2} \approx \frac{\sqrt{2 c}}{\mu \sqrt{r}}\left[\frac{\sqrt{c_{+}+c} \sqrt{c_{-}+c}}{c^{2} \sqrt{1+c} \sqrt{c_{+} c_{-}}} \mathrm{P}_{3}^{(+)} K_{I}-\frac{\mathrm{P}_{12}^{(-)}}{c^{2}} K_{I I} \operatorname{sgn}(\phi)\right] \\
& \sin \psi \\
& \quad+\frac{\sqrt{c}}{\mu \sqrt{2 r}} \frac{\mathrm{B}^{(-)} K_{I I I}}{\sqrt{1+c}} \operatorname{sgn}(\phi) \cos \psi \\
& \dot{u}_{3} \approx \frac{\sqrt{2 c}}{\mu \sqrt{r}}\left[\frac{\sqrt{c_{+}+c} \sqrt{c_{-}+c}}{c^{2} \sqrt{1+c} \sqrt{c_{+} c_{-}}} \mathrm{P}_{3}^{(-)} K_{I} \operatorname{sgn}(\phi)-\frac{\mathrm{P}_{12}^{(+)}}{c^{2}} K_{I I}\right]
\end{aligned}
$$

In (47), $\left(|\psi|<\frac{1}{2} \pi,|\phi|<\pi\right)$ and

$$
\begin{aligned}
\mathrm{P}_{3}^{(+)} & =\frac{k}{2}\left(\frac{C_{+}}{a_{-}} A_{-}^{(+)}-\frac{C_{-}}{a_{+}} A_{+}^{(+)}\right)+b \Omega \mathrm{B}^{(+)}, \\
\mathrm{P}_{3}^{(-)} & =\frac{k}{2}\left(C_{+} A_{-}^{(-)}-C_{-} A_{+}^{(-)}\right)+\Omega \mathrm{B}^{(-)}, \\
\mathrm{P}_{12}^{(+)} & =C_{+} a_{+} A_{-}^{(+)}-C_{-} a_{-} A_{+}^{(+)}+\frac{k m_{C}}{2 b} \mathrm{~B}^{(+)}, \\
\mathrm{P}_{12}^{(-)} & =C_{+} a_{+} A_{-}^{(-)}-C_{-} a_{-} A_{+}^{(-)}+\frac{k}{2} m_{C} \mathrm{~B}^{(-)} .
\end{aligned}
$$

For $\left(r \rightarrow 0,|\psi|<\frac{1}{2} \pi,|\phi|<\pi\right)$ temperature change takes the form

$$
\begin{gathered}
\theta \approx \frac{\varepsilon}{\mu \lambda} \frac{\sqrt{2 c}}{\alpha_{D} \sqrt{r}}\left[\mathrm{~T}_{12}^{(-)} \frac{K_{I I}}{c^{2}} \operatorname{sgn}(\phi)-\mathrm{T}_{3}^{(+)} \frac{\sqrt{c_{+}+c} \sqrt{c_{-}+c}}{c^{2} \sqrt{c_{+} c_{-}} \sqrt{1+c}} K_{I}\right] \\
\mathrm{T}_{12}^{(-)}=a_{-} A_{+}^{(-)}-a_{+} A_{-}^{(-)}, \quad \mathrm{T}_{3}^{(+)}=\frac{k}{2}\left(\frac{A_{+}^{(+)}}{a_{+}}-\frac{A_{-}^{(+)}}{a_{-}}\right) .
\end{gathered}
$$

\section{Criterion: dynamic energy release rate}

A standard criterion for brittle fracture, e.g., [Freund 1972], equates the rate at which surface energy is released to the rate of work associated with traction and relative displacements in the fracture zone $\mathfrak{T}$. In this study heat is neither added to, nor extracted from, the solid. Therefore, if kinetic energy is included [Gdoutos 1993] the equation takes the form

$$
D \iint_{\delta A} e_{F} \mathrm{~d} x_{1}^{0} \mathrm{~d} x_{2}^{0}=\iint_{\mathfrak{T}} \sigma_{3 k}^{C} \dot{\Delta}_{k} \mathrm{~d} x_{1}^{0} \mathrm{~d} x_{2}^{0}+D \iiint_{123} \frac{1}{2} \rho \dot{u}_{k} \dot{u}_{k} \mathrm{~d} x_{1}^{0} \mathrm{~d} x_{2}^{0} \mathrm{~d} x_{3}^{0} .
$$

Here $e_{F}$ is the surface energy per unit area, and is generally assumed to be constant [de Boer et al. 1988; Skriver and Rosengaard 1992]. Fracture zone $\mathfrak{T}$ is a strip of infinitesimal thickness in the $x_{1}^{0} x_{2}^{0}$-plane that straddles the portion of $C$ that borders $\delta A$. Subscript 123 signifies integration over the solid. Use of transport theory [Malvern 1969] and translating basis $\boldsymbol{x}$ expressed in terms of $\left(x, \psi, x_{3}=0\right)$ gives for the first term in (47)

$$
V e_{F} s \int_{\Psi} \mathrm{d} \psi c \sqrt{c^{2}+\left(c^{\prime}\right)^{2}}, \quad f^{\prime}=\frac{\mathrm{d} f}{\mathrm{~d} \psi}
$$


Here $\Psi$ signifies integration over $|\psi|<\frac{1}{2} \pi$. Use of $x$ for the integration over $\mathfrak{T}$ gives

$$
\int_{\Psi} \mathrm{d} \psi \int_{c s-}^{c s+}\left|x_{0}\right| \sigma_{3 k}^{C} \dot{\Delta}_{k} \mathrm{~d} x_{0}
$$

In light of (38) and (40) it can be shown [Freund 1972] that the integrand of (52a) features Dirac function $\delta\left(x_{0}-c s\right)$. Moreover, linear behavior in s displayed in (50) places a restriction on $\partial_{S} P_{k}$, e.g., [Achenbach and Brock 1973]. That is, $V$ must in general vary with time. One case, however, for which timeinvariance is valid is

$$
\partial_{S} P_{k}=p_{k} \sqrt{s}
$$

Equation (41) and (52a) then give

$$
\begin{aligned}
\pi \frac{s}{\mu} \int_{\Psi} V \mathrm{~d} \psi\left[\frac{R_{3}}{c^{2}} N_{12} m_{C} K_{I I}^{2}+\sqrt{\frac{1-c}{1+c}}\left(K_{I I I}^{2}+\Omega \frac{R_{3}}{c^{2}} \frac{\sqrt{c_{+}+c} \sqrt{c_{-}+c}}{\sqrt{c_{+}-c} \sqrt{c_{-}-c}} K_{I}^{2}\right)\right], \\
K_{I}=\frac{c^{2}}{g_{3}^{+} R_{3}}\left(1-\frac{c}{c_{3}}\right) p_{3}, \\
K_{I I}=\frac{c^{2}}{g_{3}^{+} R_{3}}\left(1-\frac{c}{c_{3}}\right)\left(p_{1} \cos \psi+p_{2} \sin \psi\right), \\
K_{I I I}=p_{1} \sin \psi-p_{2} \cos \psi .
\end{aligned}
$$

Equation (47) is singular near $C$. The last integration in (50) can then be, via transport theory [Malvern 1969], taken over the surface of a tube of radius $r_{C} \rightarrow 0$ that encloses the portion of $C$ that borders $\delta A$. Integration can be performed with coordinates (43) and expressions (47) and (53), so that the last term in $(50)$ becomes

$$
-\frac{s}{\mu} \int_{\Psi} V \mathrm{~d} \psi \sqrt{c^{2}+\left(c^{\prime}\right)^{2}}\left[\left(1+\frac{c}{c_{+}}\right)\left(1+\frac{c}{c_{-}}\right) \frac{K_{I}^{2} \mathrm{E}_{I}}{c^{3}(1+c)}+\frac{K_{I I}^{2}}{c^{3}} \mathrm{E}_{I I}+\frac{K_{I I I}^{2} \mathrm{E}_{I I I}}{c(1+c)}\right] .
$$

Coefficients $\left(\mathrm{E}_{I}, \mathrm{E}_{I I}, \mathrm{E}_{I I I}\right)$ are defined, in light of (48), as

$$
\begin{aligned}
\mathrm{E}_{I} & =\int_{\Psi} \cos \phi\left[\left(\mathrm{P}_{3}^{(+)}\right)^{2}+\left(\mathrm{P}_{3}^{(-)}\right)^{2}\right] \mathrm{d} \phi, \\
\mathrm{E}_{I I} & =\int_{\Psi} \cos \phi\left[\left(\mathrm{P}_{12}^{(+)}\right)^{2}+\left(\mathrm{P}_{12}^{(-)}\right)^{2}\right] \mathrm{d} \phi, \\
\mathrm{E}_{I I I} & =\int_{\Psi} \cos \phi\left(\mathrm{B}^{(-)}\right)^{2} \mathrm{~d} \phi .
\end{aligned}
$$

Here $\Phi$ signifies integration over range $|\phi|<\pi$. Equations (51), (52c) and (54) all involve integration with respect to $\psi$, so that (50) gives for $|\psi|<\frac{1}{2} \pi$,

$$
\begin{aligned}
\mu e_{F} \sqrt{c^{2}+\left(c^{\prime}\right)^{2}}= & \frac{K_{I}^{2}}{1+c}\left(1+\frac{c}{c_{+}}\right)\left(1+\frac{c}{c_{-}}\right)\left[\frac{\pi b \Omega R_{3}}{c^{2} a_{+} a_{-}}-\frac{\mathrm{E}_{I}}{c^{3}} \sqrt{c^{2}+\left(c^{\prime}\right)^{2}}\right] \\
& +K_{I I}^{2}\left[\frac{\pi R_{3}}{c^{2}} N_{12} m_{C}-\frac{\mathrm{E}_{I I}}{c^{3}} \sqrt{c^{2}+\left(c^{\prime}\right)^{2}}\right]+\frac{K_{I I I}^{2}}{1+c}\left[\pi b-\frac{\mathrm{E}_{I I I}}{c} \sqrt{c^{2}+\left(c^{\prime}\right)^{2}}\right]
\end{aligned}
$$




\section{Aspects of solution behavior}

Formula (56) is a nonlinear differential equation for $c(\psi)$. Asymptotic expressions preserved the singular behavior of $\left(\sigma_{3 k}^{C}, \dot{\Delta}_{k}, \dot{u}_{k}\right)$ and were therefore sufficient for derivation of (56). The singular behavior seen in asymptotic expression (49) for $\theta$ is also useful in generating a finite measure of thermal response near $C$. Norm $\|\theta\|$ is defined as

$$
\|\theta\|=\sqrt{\oint \theta^{2} \mathrm{~d} l}
$$

Equation (57) involves (49a) in a line integral for given $\psi$ taken counterclockwise about the circumference of the circle of radius $r=r_{C} \rightarrow 0$. Because $\mathrm{d} l=r \mathrm{~d} \phi$ integration is over range $|\phi|<\pi$, so that (57) gives for $|\psi|<\frac{1}{2} \pi$ the finite result

$$
\begin{gathered}
\|\theta\|=\frac{\sqrt{2 c}}{\mu c^{2}} \frac{\varepsilon}{\lambda \alpha_{D}} \sqrt{\left(1+\frac{c}{c_{+}}\right)\left(1+\frac{c}{c_{-}}\right) \frac{K_{I}^{2}}{1+c} \mathrm{~T}_{I}+K_{I I}^{2} \mathrm{~T}_{I I}} \\
\mathrm{~T}_{I}=\int_{\Phi}\left(\mathrm{T}_{3}^{(+)}\right)^{2} \mathrm{~d} \phi, \quad \mathrm{T}_{I I}=\int_{\Phi}\left(\mathrm{T}_{12}^{(-)}\right)^{2} \mathrm{~d} \phi .
\end{gathered}
$$

Equations (56) and (58) are somewhat complicated and opaque. Insight concerning $c(\psi)$ and $\|\theta\|$, based partly on analytical expressions, is possible by considering values of parameter $V(\psi)$ that are not negligible, but well below critical, i.e., $c / c_{3} \approx O\left(10^{-1}\right)$. To this end first-order expansions in $c^{2}$ for $\left(\mathrm{P}_{12}^{( \pm)}, \mathrm{P}_{3}^{( \pm)}, \mathrm{B}^{(-)}\right)$and $\left(\mathrm{T}_{3}^{(+)}, \mathrm{T}_{12}^{(-)}\right)$are developed and given by (E.1)-(E.3). Integration in (55) is performed on the basis of (E.1) and (E.2), and (56) gives for $|\psi|<\frac{1}{2} \pi$ asymptotic form

$$
\begin{gathered}
\frac{\mu e_{F}}{\pi} \sqrt{c^{2}+\left(c^{\prime}\right)^{2} \approx} \frac{p_{3}^{2}}{k_{C}}\left[1+E_{I}^{0} c \sqrt{c^{2}+\left(c^{\prime}\right)^{2}}\right] \\
+\frac{1}{k_{C}}\left(p_{1} \cos \psi+p_{2} \sin \psi\right)^{2}\left[1 / \Omega+E_{I I}^{0} c \sqrt{c^{2}+\left(c^{\prime}\right)^{2}}\right] \\
+\left(p_{1} \sin \psi-p_{2} \cos \psi\right)^{2}\left[1+E_{I I}^{0} c \sqrt{c^{2}+\left(c^{\prime}\right)^{2}}\right], \\
E_{I}^{0}=\frac{5}{32}\left(1+\frac{1}{c_{D}^{2}}\right), \quad E_{I I}^{0}=\frac{1}{32} \frac{4+3 c_{D}^{2}}{c_{D}^{2}-1}, \quad E_{I I I}^{0}=\frac{1}{4}, \\
k_{C}=2\left(1-\frac{1}{c_{D}^{2}}\right) .
\end{gathered}
$$

Use of (E.1), (E.3) and (58b) leads to an asymptotic form of (58a) for $|\psi|<\frac{1}{2} \pi$ :

$$
\|\theta\| \approx \frac{\varepsilon}{\lambda \alpha_{D}} \frac{\sqrt{33 \pi c}}{8 \mu\left(c_{D}^{2}-1\right)} \sqrt{p_{3}^{2}+\left(p_{1} \cos \psi+p_{2} \sin \psi\right)^{2}} .
$$

Equation (59a) differs from its counterpart in [Brock 2017b] in that it does depend on thermal properties, i.e., $\Omega$. In the previous work, thermal properties have a second-order, i.e., $O\left(c^{4}\right)$ effect. Equation (60) differs from its counterpart in that the relative influence of compression and shear loading depends only on $\psi$ and the values of $p_{k}$. Equation (59) and (60) are now used to study two cases. 


\section{Case A: pure compression}

Here $p_{1}=p_{2}=0, p_{3}=p_{A}>0$. Problem symmetry exists with respect to the $x_{1}^{0} x_{3}^{0}$-plane, and (59) reduces to

$$
\begin{gathered}
\left(1-c_{A}^{2} E_{I}^{0} z\right) z=1, \\
z=\frac{c}{c_{A}}, \quad c_{A}=\frac{\pi p_{A}^{2}}{\mu e_{F} k_{C}} .
\end{gathered}
$$

When kinetic energy is, respectively, neglected $\left(E_{I}^{0}=0\right)$ and included, (61a) gives

$$
\begin{aligned}
& c(\psi)=c_{A}, \\
& c(\psi)=c_{A}^{E}=\frac{1}{2 E_{I}^{0} c_{A}}\left(1-\sqrt{1-4 E_{I}^{0} c_{A}^{2}}\right) .
\end{aligned}
$$

Equation (62) describes circular crack edge extension zone contours. Equation (60) gives for (62a) and (62b) respectively the constant values

$$
\|\theta\| \approx \frac{\varepsilon}{\lambda \alpha_{D}} \frac{\sqrt{33 \pi} p_{A}}{8 \mu\left(c_{D}^{2}-1\right)}\left(\sqrt{c_{A}}, \sqrt{c_{A}^{E}}\right) .
$$

For illustration consider a generic metal with properties [de Boer et al. 1988; Skriver and Rosengaard 1992; Brock 2009; Ignaczak and Ostoja-Starzewski 2010]

$$
\begin{aligned}
& \mu=79 \mathrm{GPa}, \quad e_{F}=2.2 \mathrm{~J} / \mathrm{m}^{2}, \quad V_{S}=3094 \mathrm{~m} / \mathrm{s}, \quad c_{D}=2, \\
& c_{+}=4.5452, \quad c_{-}=1.997, \quad c_{3}=0.9332, \quad T_{0}=294 \mathrm{~K}, \\
& \alpha_{D}=89.6\left(10^{-6}\right) \mathrm{K}^{-1}, \quad \varepsilon=0.05044, \quad h=3.1862\left(10^{-9}\right) \mathrm{m}, \quad h_{0}=1.547\left(10^{-10}\right) \mathrm{m} .
\end{aligned}
$$

Calculations for $c_{A}, c_{A}^{E}$ and $\|\theta\|$ are given in Table 1 for different values of $p_{A}$. There $c_{A}^{E}>c_{A}$, thus showing that neglect of kinetic energy leads to under-prediction of crack extension speed $V \cos \psi$. This effect decreases with increasing $p_{A}$ however. Entries for $\|\theta\|$ that correspond to $\left(c_{A}, c_{A}^{E}\right)$ behave in the same manner, but the under-prediction effect is more pronounced. It is noted that the same behavior is exhibited in [Brock 2017b]. However, there the effect of increasing $p_{A}$ on the under-prediction of $\|\theta\|$ is less pronounced.

\section{Case B: mixed-mode loading}

Here $\left(p_{1}=f_{B} p_{3}, p_{2}=0, p_{3}=p_{B}\right)$ with $\left(0<f_{B}<1, p_{B}>0\right)$. Problem symmetry again exists with respect to the $x_{1}^{0} x_{3}^{0}$-plane, and (59) can now reduce for $|\psi|<\frac{1}{2} \pi$ to

$$
\begin{gathered}
\sqrt{z^{2}+\left(z^{\prime}\right)^{2}}\left[1-z\left(\Omega_{0}+\Omega_{1} \cos ^{2} \psi\right)\right]=1+f_{B}^{2} k_{C}+f_{B}^{2}\left(1 / \Omega-k_{C}\right) \cos ^{2} \psi, \\
\|\theta\|=\frac{\varepsilon p_{B}}{\lambda \alpha_{D}} \frac{\sqrt{33 \pi c(\psi)}}{8 \mu\left(c_{D}^{2}-1\right)} \sqrt{1+f_{B}^{2} \cos ^{2} \psi}, \\
z=\frac{c(\psi)}{c_{B}}, \quad c_{B}=\frac{\pi p_{B}^{2}}{\mu e_{F} k_{C}} .
\end{gathered}
$$


kinetic energy neglected

\begin{tabular}{|cccccc|}
\hline$f_{A}$ & 1 & 3 & 5 & 10 & 15 \\
\hline$c_{A}$ & 0.00127 & 0.01143 & 0.03174 & 0.12694 & 0.28562 \\
$f_{N}$ & 0.05509 & 0.49578 & 1.37714 & 5.50834 & 12.3934 \\
\hline
\end{tabular}

kinetic energy included

\begin{tabular}{|cccccc|}
\hline$f_{A}$ & 1 & 3 & 5 & 10 & 15 \\
\hline$c_{A}^{E}$ & 0.02017 & 0.01143 & 0.31741 & 0.12734 & 0.29032 \\
$f_{N}$ & 0.21957 & 0.49578 & 1.37727 & 5.51726 & 12.4959 \\
\hline
\end{tabular}

Table 1. Case A: $c_{A}, c_{A}^{F}$, $\|\theta\|=f_{N}\left(10^{-1}\right) \mathrm{Km}^{1 / 2}$ for $p_{A}=f_{A}\left(10^{4}\right) \mathrm{N} / \mathrm{m}^{3 / 2}$.

When kinetic energy is neglected we have $\Omega_{0}=\Omega_{1}=0$; when it is included,

$$
\Omega_{0}=c_{B}^{2}\left(E_{I}^{0}+k_{C} f_{B}^{2} E_{I I}^{0}\right), \quad \Omega_{1}=c_{B}^{2} f_{B}^{2}\left(E_{I I}^{0}-k_{C} E_{I I I}^{0}\right) .
$$

Explicit $\psi$-dependence of (64a) implies that the crack extension zone contour is not a circular arc, and that obtaining an analytical solution for $c(\psi)$ may not be simple. Analysis in [Brock 2017b] suggests use of series representation:

$$
c(\psi)=c_{B}\left[g_{0}+\sum_{1}^{N} \frac{g_{2 j}}{2 j} \cos ^{2 j} \psi\right] .
$$

Substitution of (66) into (64a) and equating coefficients of terms $\cos ^{2 j} \psi$ gives recursive equations for $\left(g_{0}, g_{2 j}\right)$. Equations for $\left(g_{0}, g_{2}\right)$ are quadratic, and solutions are given in Appendix F. Equations for $\left(g_{4}, g_{6}, \ldots\right)$ are linear.

Calculations for $c$ and $\|\theta\|$ are listed in Table 2 (kinetic energy neglected) and Table 3 (kinetic energy included) for various values of $\psi$ and loading ratio $f_{B}$. Compression load $p_{3}=p_{B}=5\left(10^{4}\right) \mathrm{N} / \mathrm{m}^{1 / 2}$ is used, and the same generic metal featured in Table 1. Entries for $c$ show that increasing $f_{B}$ (i.e., relative importance of shear loading) produces crack contours that are somewhat elliptical. That is the maximum rate of crack extension into the solid is less than the rate at which new crack surface spreads along the original, semi-infinite crack contour. Inclusion of kinetic energy appears to enhance the deviation from a circular arc. It is also noted that the relation between the two rates is the reverse of that found in [Brock $2017 \mathrm{~b}]$, where discontinuity in temperature across the crack plane is not allowed.

Entries in Table 2 and Table 3 indicate that increasing $f_{B}$ also enhances $\|\theta\|$. When kinetic energy is neglected the maximum value occurs directly ahead of the translating point forces. When kinetic energy is included however, $\|\theta\|$ can achieve maximum values for $|\psi| \neq 0$ when $f_{B}$ is large enough. A maximum for $|\psi| \neq 0$ is also seen in [Brock 2017b], which however only considered the case $p_{1}=p_{3}$.

\section{Some observations}

This paper extends the range of [Brock $2017 \mathrm{a} ; 2017 \mathrm{~b}$ ] for 3D dynamic fracture by considering a transient problem with mixed-mode loading in a thermoelastic solid with relaxation. The solid is initially at rest 
kinetic energy neglected

\begin{tabular}{|c|lllll|}
\hline & $f_{B}=\frac{1}{4}$ & $f_{B}=\frac{1}{3}$ & $f_{B}=\frac{1}{2}$ & $f_{B}=\frac{2}{3}$ & $f_{B}=1.0$ \\
\hline$\psi=0^{\circ}: c$ & 0.03417 & 0.03605 & 0.04136 & 0.04881 & 0.06522 \\
$f_{N}$ & 1.47304 & 1.54726 & 1.7578 & 2.0526 & 2.79198 \\
\hline$\psi=15^{\circ}: c$ & 0.03421 & 0.03612 & 0.0 .0415 & 0.04906 & 0.0663 \\
$f_{N}$ & 1.47091 & 1.54344 & 1.74908 & 2.03649 & 2.76738 \\
\hline$\psi=30^{\circ}: c$ & 0.03431 & 0.03629 & 0.04191 & 0.04975 & 0.06914 \\
$f_{N}$ & 1.46503 & 1.53287 & 1.72458 & 1.99105 & 2.689 \\
\hline$\psi=45^{\circ}: c$ & 0.03444 & 0.03654 & 0.04248 & 0.05075 & 0.07279 \\
$f_{N}$ & 1.45689 & 1.51811 & 1.68996 & 1.92525 & 2.5544 \\
\hline$\psi=60^{\circ}: c$ & 0.03458 & 0.03678 & 0.04305 & 0.05179 & 0.07619 \\
$f_{N}$ & 1.44862 & 1.05301 & 1.65333 & 1.8545 & 2.38568 \\
\hline$\psi=75^{\circ}: c$ & 0.34674 & 0.03696 & 0.04348 & 0.05259 & 0.07852 \\
$f_{N}$ & 1.4425 & 1.49167 & 1.62539 & 1.79905 & 2.23758 \\
\hline$\psi=90^{\circ}: c$ & 0.03471 & 0.03702 & 0.04364 & 0.05209 & 0.07934 \\
$f_{N}$ & 1.44024 & 1.48747 & 1.61492 & 1.77788 & 2.17748 \\
\hline
\end{tabular}

Table 2. Case B: $c,\|\theta\|=f_{N}\left(10^{-6}\right) \mathrm{Km}^{1 / 2}$ for $p_{B}=5\left(10^{4}\right) \mathrm{N} / \mathrm{m}^{3 / 2}$, various $f_{B}=$ $p_{1} / p_{B}$ and $\psi$.

at uniform (absolute) temperature, and contains a semi-infinite, closed slit. Shearing and compressive point forces are applied just behind the crack edge, and initiate brittle fracture. Dynamic similarity is assumed, i.e., extension rate of points on the crack edge is constant in time, but can vary with location.

Unilateral temporal and spatial transforms are employed. In the latter case however, use is made of variable transformations based on quasipolar coordinates. Focus upon fracture initiation, moreover, justifies use of asymptotic expressions that, in integral transform space, give four equations that relate discontinuity in crack surface temperature, crack opening, crack plane heat flux and traction. The equations can be rewritten in Wiener-Hopf [Morse and Feshbach 1953; Achenbach 1976] form. Analytical solutions for transforms with inverse valid near the crack edge are obtained. Such inverses are sufficient to derive the nonlinear differential equation for the crack edge contour and an exact formula for the norm of the change in crack edge temperature.

As is predictable [Achenbach and Brock 1973], assumption of dynamic similarity restricts the type of time variation of the point forces. A suitable type is identified, however, and used to study an example of pure compression (Case A) and one of mixed-mode loading (Case B). For Case A, the extending portion of the crack edge is circular, and the norm of temperature near the edge is constant. In Case $\mathrm{B}$, extending portion of the crack edge is elliptical, with the maximum rate of extension into the solid being less than the expansion rate of new crack surface along the original rectilinear crack contour. The temperature norm is also not constant. In both cases, inclusion of kinetic energy gives larger extension rates and temperature norms. However, this effect decreases as the force magnitudes are increased. 
kinetic energy included

\begin{tabular}{|c|lllll|}
\hline & $f_{B}=\frac{1}{4}$ & $f_{B}=\frac{1}{3}$ & $f_{B}=\frac{1}{2}$ & $f_{B}=\frac{2}{3}$ & $f_{B}=1.0$ \\
\hline$\psi=0^{\circ}: c$ & 0.03414 & 0.0361 & 0.04172 & 0.04843 & 0.05253 \\
$f_{N}$ & 1.47228 & 1.54833 & 1.76545 & 2.04463 & 2.5057 \\
\hline$\psi=15^{\circ}: c$ & 0.03422 & 0.03616 & 0.04183 & 0.05063 & 0.05526 \\
$f_{N}$ & 1.47116 & 1.5444 & 1.75575 & 2.06896 & 2.5266 \\
\hline$\psi=30^{\circ}: c$ & 0.03432 & 0.03632 & 0.04213 & 0.05078 & 0.06204 \\
$f_{N}$ & 1.4652 & 1.53346 & 1.72904 & 2.01157 & 2.5473 \\
\hline$\psi=45^{\circ}: c$ & 0.03444 & 0.03648 & 0.04258 & 0.05122 & 0.0697 \\
$f_{N}$ & 1.45696 & 1.51838 & 1.69195 & 1.9342 & 2.4996 \\
\hline$\psi=60^{\circ}: c$ & 0.03458 & 0.03678 & 0.04309 & 0.05194 & 0.07551 \\
$f_{N}$ & 1.44864 & 1.50301 & 1.65411 & 1.85707 & 2.37501 \\
\hline$\psi=75^{\circ}: c$ & 0.03467 & 0.03696 & 0.0435 & 0.05263 & 0.07858 \\
$f_{N}$ & 1.4425 & 1.49169 & 1.62577 & 1.79968 & 2.23839 \\
\hline$\psi=90^{\circ}: c$ & 0.03471 & 0.03702 & 0.04365 & 0.05293 & 0.07945 \\
$f_{N}$ & 1.44024 & 1.48747 & 1.61516 & 1.77844 & 2.17899 \\
\hline
\end{tabular}

Table 3. Case B: $c,\|\theta\|=f_{N}\left(10^{-6}\right) \mathrm{Km}^{1 / 2}$ for $p_{B}=5\left(10^{4}\right) \mathrm{N} / \mathrm{m}^{3 / 2}$, various $f_{B}=$ $p_{1} / p_{B}$ and $\psi$.

Analysis considers the Lord and Shulman [1967] thermal relaxation model. Sub-Rayleigh crack extension rates are treated and, in contrast to [Brock 2017b], thermoelastic properties affect both the rates and crack edge temperature norm. The difference represents the inclusion of temperature discontinuity. Another difference with [Brock 2017b] is that the role of compression/shear ratio in mixed-mode loading is examined here.

\section{Appendix A}

$$
\begin{aligned}
& U_{1}^{( \pm)}=\frac{q B}{\beta^{2}} \bar{\Delta}_{3} \cos \psi-\frac{1}{2 B \beta^{2}}\left(B^{2} \frac{\bar{\Sigma}_{1}}{\mu p}+q^{2} \frac{\bar{\Sigma}_{M}}{\mu p} \sin \psi\right) \\
& \quad(\mp) \frac{q}{2 \beta^{2}} \frac{\bar{\Sigma}_{3}}{\mu p} \cos \psi( \pm) \frac{1}{2 \beta^{2}}\left(T \bar{\Delta}_{1}+2 q^{2} \bar{\Delta}_{M} \sin \psi\right) \\
& U_{2}^{( \pm)}=\frac{q B}{\beta^{2}} \bar{\Delta}_{3} \sin \psi-\frac{1}{2 B \beta^{2}}\left(B^{2} \frac{\bar{\Sigma}_{2}}{\mu p}-q^{2} \frac{\bar{\Sigma}_{M}}{\mu p} \cos \psi\right) \\
& \quad(\mp) \frac{q}{2 \beta^{2}} \frac{\bar{\Sigma}_{3}}{\mu p} \sin \psi( \pm) \frac{1}{2 \beta^{2}}\left(T \bar{\Delta}_{2}-2 q^{2} \bar{\Delta}_{M} \cos \psi\right) \\
& U_{+}^{( \pm)}=\frac{1}{2 \Omega \beta^{2} A_{+}}\left[\frac{\alpha_{D}}{p^{2}} \mathrm{~d} \bar{\Theta}+C_{-}\left(T \bar{\Delta}_{3}+q \bar{\Sigma}_{P}\right)\right](\mp) \frac{1}{2 \Omega \beta^{2}}\left[\frac{\alpha_{D}}{p} \bar{\Theta}+C_{-}\left(\frac{\bar{\Sigma}_{3}}{\mu p}+2 q \bar{\Delta}_{P}\right)\right]
\end{aligned}
$$




$$
\begin{array}{cc}
U_{-}^{( \pm)}=\frac{-1}{2 \Omega \beta^{2} A_{-}}\left[\frac{\alpha_{D}}{p^{2}} \mathrm{~d} \bar{\Theta}+C_{+}\left(T \bar{\Delta}_{3}+q \bar{\Sigma}_{P}\right)\right]( \pm) \frac{1}{2 \Omega \beta^{2}}\left[\frac{\alpha_{D}}{p} \bar{\Theta}+C_{+}\left(\frac{\bar{\Sigma}_{3}}{\mu p}+2 q \bar{\Delta}_{P}\right)\right], \\
T=\beta^{2}-2 q^{2} . \\
\bar{\Delta}_{P}=\bar{\Delta}_{1} \cos \psi+\bar{\Delta}_{2} \sin \psi, & \bar{\Delta}_{M}=\bar{\Delta}_{1} \sin \psi-\bar{\Delta}_{2} \cos \psi, \\
\bar{\Sigma}_{P}=\bar{\Sigma}_{1} \cos \psi+\bar{\Sigma}_{2} \sin \psi, & \bar{\Sigma}_{M}=\bar{\Sigma}_{1} \sin \psi-\bar{\Sigma}_{2} \cos \psi .
\end{array}
$$

\section{Appendix B}

$$
\begin{aligned}
\frac{1}{\mu}\left(\bar{\sigma}_{31}^{C}-\frac{P_{1}}{p \beta}\right) & =\frac{\cos \psi}{\Omega \beta^{2}}\left(\frac{p M_{12}}{2 B} \bar{\Delta}_{P}+q M_{A} \alpha_{D} \bar{\Theta}\right)+p B \bar{\Delta}_{M} \sin \psi \\
\frac{1}{\mu}\left(\bar{\sigma}_{32}^{C}-\frac{P_{2}}{p \beta}\right) & =\frac{\sin \psi}{\Omega \beta^{2}}\left(\frac{p M_{12}}{2 B} \bar{\Delta}_{P}+q M_{A} \alpha_{D} \bar{\Theta}\right)-p B \bar{\Delta}_{M} \cos \psi \\
\frac{1}{\mu}\left(\bar{\sigma}_{33}^{C}-\frac{P_{3}}{p \beta}\right) & =\frac{p B}{2 A_{+} A_{-}} \frac{M_{3} \bar{\Delta}_{3}}{\Omega \beta^{2}} . \\
\partial_{3} \bar{\theta}^{C} & =\frac{\varepsilon}{\alpha_{D} \Gamma_{+} \Gamma_{-}} p^{2} q M_{A} \bar{\Delta}_{P}-\frac{p}{2 \Omega} M_{C} \bar{\Theta} \\
M_{12} & =C_{-} M_{+}-C_{+} M_{-}, \\
M_{3} & =C_{-} \frac{A_{-}}{B} M_{+}-C_{+} \frac{A_{+}}{B} M_{-}, \\
M_{A} & =A_{+}-A_{-}, \quad M_{C}=C_{+} A_{+}-C_{-} A_{-}, \\
M_{ \pm} & =T^{2}+4 q^{2} A_{ \pm} B .
\end{aligned}
$$

\section{Appendix C}

$$
\begin{aligned}
& G_{3}^{ \pm}(q)=\exp \left[\frac{1}{\pi} \int \frac{\mathrm{d} u}{u \mp c} \frac{S_{3}(u)}{q(u \mp c) \pm 1}\right] \quad\left(1<u<c_{+}\right), \\
& S_{3}(u)=-\tan ^{-1} \frac{1}{4 \Omega} \frac{k^{2}(u) m_{C}(u)}{a_{+}(u) a_{-}(u) \sqrt{u^{2}-1}} \quad\left(1<u<c_{-}\right), \\
& S_{3}(u)=\tan ^{-1} \frac{a_{+}(u)}{C_{-}}\left[4 \Omega \frac{\sqrt{u^{2}-1}}{k^{2}(u)}-\frac{c_{-} C_{+}}{\sqrt{u^{2}-c_{-}^{2}}}\right] \quad\left(c_{-}<c<c_{+}\right) . \\
& G_{12}^{ \pm}(q)=\exp \left[\frac{1}{\pi} \int \frac{\mathrm{d} u}{u \mp c} \frac{S_{12}(u)}{q(u \mp c) \pm 1}\right] \quad\left(1<u<c_{+}\right), \\
& S_{12}(u)=\tan ^{-1} \frac{4}{\Omega} \frac{n_{C}(u)}{k^{2}(u)} \sqrt{u^{2}-1} \quad\left(1<u<c_{-}\right), \\
& n_{C}(u)=C_{-} a_{+}(u)-C_{+} a_{-}(u)
\end{aligned}
$$




$$
\begin{gathered}
S_{12}(u)=\tan ^{-1} \frac{C_{-} a_{+}(u)}{\frac{\Omega}{4} \frac{k^{2}(u)}{\sqrt{u^{2}-1}}-\frac{C_{+}}{c_{-}} \sqrt{u^{2}-c_{-}^{2}}}\left(c_{-}<u<c_{+}\right) . \\
G_{A}^{ \pm}(q)=\exp \left[\frac{1}{\pi} \int \frac{1}{u \mp c} \tan ^{-1} \frac{\sqrt{u^{2}-c_{-}^{2}}}{c_{-} a_{+}(u)} \frac{\mathrm{d} u}{q(u \mp c) \pm 1}\right] \quad\left(c_{-}<u<c_{+}\right) . \\
G_{C}^{ \pm}(q)=\exp \left[\frac{1}{\pi} \int \frac{1}{u \mp c} \tan ^{-1} \frac{C_{-} \sqrt{u^{2}-c_{-}^{2}}}{c_{-} C_{+} a_{+}(u)} \frac{\mathrm{d} u}{q(u \mp c) \pm 1}\right] \quad\left(c_{-}<u<c_{+}\right) .
\end{gathered}
$$

\section{Appendix D}

Asymptotic $(|q| \rightarrow \infty)$ expressions with inverses valid for $x_{3}=0, x \rightarrow 0+$ :

$$
\alpha_{D} \partial_{3} \bar{\theta}^{C} \approx-\frac{\varepsilon \sqrt{q}}{\mu \Gamma_{+} \Gamma_{-}} \frac{4 c^{2} m_{A} m_{C}}{g_{3}^{+} R_{3} \sqrt{c}}\left(1-\frac{c}{c_{3}}\right)\left(P_{1} \cos \psi+P_{2} \sin \psi\right)
$$

$$
\begin{gathered}
\bar{\sigma}_{31}^{C} \approx-\frac{\sin \psi}{p \sqrt{q}} \frac{\sqrt{1-c}}{\sqrt{c}}\left(P_{1} \sin \psi-P_{2} \cos \psi\right)-\frac{\cos \psi}{p \sqrt{q}} \frac{N_{12}}{g_{3}^{+} \sqrt{c}}\left(1-\frac{c}{c_{3}}\right)\left(P_{1} \cos \psi+P_{2} \sin \psi\right), \\
\bar{\sigma}_{32}^{C} \approx-\frac{\cos \psi}{p \sqrt{q}} \frac{\sqrt{1-c}}{\sqrt{c}}\left(P_{1} \sin \psi-P_{2} \cos \psi\right)-\frac{\sin \psi}{p \sqrt{q}} \frac{N_{12}}{g_{3}^{+} \sqrt{c}}\left(1-\frac{c}{c_{3}}\right)\left(P_{1} \cos \psi+P_{2} \sin \psi\right), \\
\bar{\sigma}_{33}^{C} \approx \frac{P_{3}}{p \sqrt{q}} \frac{1}{g_{3}^{+}}\left(1-\frac{c}{c_{3}}\right) \frac{\sqrt{c_{+}-c_{-}} \sqrt{1-c}}{\sqrt{c} \sqrt{c_{+}-c_{-}} \sqrt{c_{-}-c}} . \\
N_{12}=1+\frac{8 \varepsilon}{\lambda} \frac{m_{A}^{2}}{R_{3}}
\end{gathered}
$$

Asymptotic $(|q| \rightarrow \infty)$ expressions with inverses valid for $x_{3}=0, x \rightarrow 0-$ :

$$
\alpha_{D} \bar{\Theta} \approx-\frac{4 \varepsilon}{\Gamma_{+} \Gamma_{-}} \frac{m_{A}}{\mu p \sqrt{-q}} \frac{c^{2}}{\sqrt{c} g_{3}^{+} R_{3}}\left(1-\frac{c}{c_{3}}\right)\left(P_{1} \cos \psi+P_{2} \sin \psi\right)
$$

$$
\begin{aligned}
p \beta \bar{\Delta}_{1} \approx-\frac{2 \sin \psi}{\mu p \sqrt{-q}} \frac{\sqrt{c}}{\sqrt{1+c}}\left(P_{1} \sin \psi-P_{2} \cos \psi\right) & \\
& -\frac{2 \cos \psi}{\mu p \sqrt{-q}} \frac{c^{2} \sqrt{c}}{g_{3}^{+} R_{3}}\left(1-\frac{c}{c_{3}}\right)\left(P_{1} \cos \psi+P_{2} \sin \psi\right),
\end{aligned}
$$

$$
\begin{aligned}
p \beta \bar{\triangle}_{2} \approx-\frac{2 \cos \psi}{\mu p \sqrt{-q}} \frac{\sqrt{c}}{\sqrt{1+c}}\left(P_{1} \sin \psi-P_{2} \cos \psi\right) & -\frac{2 \sin \psi}{\mu p \sqrt{-q}} \frac{c^{2} \sqrt{c}}{g_{3}^{+} R_{3}}\left(1-\frac{c}{c_{3}}\right)\left(P_{1} \cos \psi+P_{2} \sin \psi\right), \\
p \beta \bar{\triangle}_{3} \approx & \frac{2 P_{3}}{\mu p \sqrt{-q}} \frac{\Omega c^{2}}{g_{3}^{+} R_{3}}\left(1-\frac{c}{c_{3}}\right) \frac{\sqrt{c}}{\sqrt{c_{+} c_{-}} \sqrt{1+c}} \sqrt{c_{+}+c} \sqrt{c_{-}+c}
\end{aligned}
$$




\section{Appendix E}

$$
\begin{gathered}
\mathrm{A}_{ \pm}^{(+)} \approx \sqrt{1+\cos \phi}\left[1+\frac{c^{2}}{4 c_{ \pm}^{2}}(1-\cos \phi) \cos \phi\right] \\
\mathrm{A}_{ \pm}^{(-)} \approx \sqrt{1-\cos \phi}\left[1-\frac{c^{2}}{4 c_{ \pm}^{2}}(1+\cos \phi) \cos \phi\right] \\
\mathrm{B}^{(+)} \approx \sqrt{1+\cos \phi}\left[1+\frac{1}{4} c^{2}(1-\cos \phi) \cos \phi\right] \\
\mathrm{B}^{(-)} \approx \sqrt{1-\cos \phi}\left[1-\frac{1}{4} c^{2}(1+\cos \phi) \cos \phi\right] \\
\mathrm{P}_{3}^{(+)} \approx-\frac{\Omega c^{2}}{2 c_{D}^{2}} \sqrt{1+\cos \phi}\left[1-\frac{1}{2} \cos \phi\left(c_{D}^{2}-1\right)(1-\cos \phi)\right] \\
\mathrm{P}_{3}^{(-)} \approx \frac{\Omega c^{2}}{2 c_{D}^{2}} \sqrt{1-\cos \phi}\left[c_{D}^{2}+\frac{1}{2} \cos \phi\left(1-c_{D}^{2}\right)(1+\cos \phi)\right] \\
\mathrm{P}_{12}^{(+)} \approx \frac{\Omega c^{2}}{4 c_{D}^{2}} \sqrt{1+\cos \phi} \cos \phi(1-\cos \phi)\left(c_{D}^{2}-1\right), \\
\mathrm{P}_{12}^{(-)} \approx-\frac{\Omega c^{2}}{2 c_{D}^{2}} \sqrt{1-\cos \phi}\left[c_{D}^{2}+\frac{1}{2} \cos \phi(1+\cos \phi)\left(c_{D}^{2}-1\right)\right] \\
\mathrm{T}_{3}^{(+)} \approx \frac{\Omega c^{2}}{2 c_{D}^{2}} \sqrt{1+\cos \phi}\left[1+\frac{1}{2} \cos \phi(1-\cos \phi)\right] \\
\mathrm{T}_{12}^{(-)} \approx-\frac{\Omega c^{2}}{2 \lambda_{D}^{2}} \sqrt{1-\cos \phi}\left[1-\frac{1}{2} \cos \phi(1+\cos \phi)\right]
\end{gathered}
$$

\section{Appendix $\mathbf{F}$}

Kinetic energy neglected:

$$
\begin{aligned}
& g_{0}=1+f_{B}^{2} k_{C} \\
& g_{2}=-\frac{1}{2} g_{0}\left[1-\sqrt{1+2 / g_{0}\left(2 f_{B}\right)^{2}\left(1 / \Omega-k_{C}\right)}\right]
\end{aligned}
$$

Kinetic energy included:

$$
\begin{aligned}
& g_{0}=\frac{1}{2 \Omega_{0}}\left(1-C_{B}\right), \quad C_{B}=\sqrt{1-4 \Omega_{0}\left(1+f_{B}^{2} k_{C}\right)}, \\
& g_{2}=-2 F_{1} / F_{2}^{2}\left[1-\sqrt{1-F_{0}\left(F_{2} / F_{1}\right)^{2}}\right] . \\
& F_{0}=\Omega_{1} g_{0}^{3}\left(2-C_{B}\right)-2 f_{B}^{2}\left(1 / \Omega-k_{C}\right)\left(1+f_{B}^{2} k_{C}\right), \\
& F_{1}=\left(1+f_{B}^{2} k_{C}\right) C_{B}, \quad F_{2}=1+C_{B} .
\end{aligned}
$$




\section{References}

[Abramowitz and Stegun 1972] M. Abramowitz and I. A. Stegun (editors), Handbook of mathematical functions, Dover, New York, 1972.

[Achenbach 1976] J. D. Achenbach, Wave propagation in elastic solids, 1st ed., North-Holland Series in Applied Mathematics and Mechanics 16, North-Holland Publishing Co., Amsterdam, 1976.

[Achenbach and Brock 1973] J. D. Achenbach and L. M. Brock, "On quasistatic and dynamic fracture", pp. 529-541 in Proceedings of an international conference on dynamic crack propagation, edited by G. C. Sih, Springer, Dordrecht, 1973.

[Barber 1992] J. R. Barber, Elasticity, Solid Mechanics and its Applications 12, Kluwer Academic Publishers Group, Dordrecht, The Netherlands, 1992.

[de Boer et al. 1988] F. R. de Boer, W. C. M. Mattens, R. Boom, A. R. Miedema, and A. K. Niessen, Cohesion in metals, North-Holland, Amsterdam, 1988.

[Brock 2009] L. M. Brock, "Basic problems of coupled thermoelasticity with thermal relaxation and pre-stress: aspects observed in exact and asymptotic solutions", J. Therm. Stresses 32:6-7 (2009), 593-622.

[Brock 2012] L. M. Brock, "Two cases of rapid contact on an elastic half-plane: the sliding ellipsoid, rolling sphere", J. Mech. Mater: Struct. 7:5 (2012), 469-483.

[Brock 2017a] L. Brock, "Transient growth of a planar crack in three dimensions: mixed mode", J. Mech. Mater. Struct. 12:3 (2017), 313-328.

[Brock 2017b] L. M. Brock, "Fracture initiation in a thermoelastic solid with relaxation: transient three-dimensional analysis", J. Therm. Stresses 40:8 (2017), 995-1014.

[Brock and Achenbach 1973] L. Brock and J. Achenbach, "Extension of an interface flaw under the influence of transient waves", Int. J. Solids Struct. 9 (1973), 53-68.

[Freund 1972] L. B. Freund, "Energy flux into the tip of an extending crack in an elastic solid", J. Elasticity 2:4 (1972), 341-349.

[Freund 1990] L. B. Freund, Dynamic fracture mechanics, Cambridge University Press, 1990.

[Gdoutos 1993] E. E. Gdoutos, Fracture mechanics: an introduction, Solid Mechanics and its Applications 14, Kluwer Academic Publishers Group, Dordrecht, The Netherlands, 1993.

[Ignaczak and Ostoja-Starzewski 2010] J. Ignaczak and M. Ostoja-Starzewski, Thermoelasticity with finite wave speeds, Oxford University Press, 2010.

[Lord and Shulman 1967] H. W. Lord and Y. Shulman, "A generalized dynamical theory of thermoelasticity", J. Mech. Phys. Solids 15:5 (1967), 299-309.

[Malvern 1969] L. S. Malvern, Introduction to the mechanics of continuous media, Prentice-Hall, Englewood Cliffs, NJ, 1969. [Morse and Feshbach 1953] P. M. Morse and H. Feshbach, Methods of theoretical physics, McGraw-Hill, New York, 1953.

[Skriver and Rosengaard 1992] H. L. Skriver and N. M. Rosengaard, "Surface energy and work function of elemental metals", Phys. Rev. B 46 (1992), 7157-7168.

[Sneddon 1972] I. N. Sneddon, The use of integral transforms, McGraw-Hill, New York, 1972.

Received 28 Apr 2017. Accepted 17 May 2017.

LouIS M. BROCK: louis . brock@uky . edu

Department of Mechanical Engineering, University of Kentucky, Lexington, KY, United States 